\title{
Alternatively spliced products lacking exon 12 dominate the expression of fragile $X$ mental retardation 1 gene in human tissues
}

\author{
XIANGUO FU ${ }^{1}$, DEZHU ZHENG ${ }^{2}$, JUAN LIAO ${ }^{2}$, QINGQIN LI $^{2}$, YUXIANG LIN ${ }^{2}$, \\ DUO ZHANG $^{2}$, AIZHEN YAN ${ }^{2}$ and FENGHUA LAN ${ }^{2}$ \\ ${ }^{1}$ Department of Pathology, School of Basic Medical Sciences, Fujian Medical University, Fuzhou, Fujian 350004; \\ ${ }^{2}$ Department of Clinical Genetics and Experimental Medicine, Fuzhou General Hospital/Clinical College of \\ Fujian Medical University, Fuzhou, Fujian 350025, P.R. China
}

Received May 9, 2014; Accepted February 17, 2015

DOI: $10.3892 / \mathrm{mmr} .2015 .3574$

\begin{abstract}
Fragile $\mathrm{X}$ mental retardation 1 gene (FMRl) expression is associated with fragile $\mathrm{X}$ syndrome (FXS) and exhibits several splicing products. However, the proportion of spliced isoforms that are expressed in different tissues remains unclear. In the present study, long-chain reverse transcription-polymerase chain reaction with a $\mathrm{T}$ cloning-sequencing method was conducted in order to analyze the entire coding region of the FMRI gene in human tissues. In particular, FXS-associated tissues were analyzed, including the brain and testis. Twenty alternatively spliced isoforms were observed among 271 recombinants, including six novel ones. The isoform that consisted of the entire FMRI coding region (ISO1) accounted for a small proportion of all isoforms. Isoforms lacking exon 12 were the most abundant. In particular, spliced isoforms ISO7 and ISO17 were the most abundant. However, their relative abundance varied between the peripheral blood cells, and the testis and brain tissues. Bioinformatic analyses suggested that exon 12 may be the sole exon undergoing positive selection. The results of the present study suggested that the mechanisms underlying alternative splicing (AS) of the FMRl gene may be more complex. Furthermore, the functions of alternatively spliced products lacking exon 12 require further investigation. The results of the present study provide novel insights into the association between AS and the structure and function of the FMRl gene.
\end{abstract}

Correspondence to: Professor Fenghua Lan, Department of Clinical Genetics and Experimental Medicine, Fuzhou General Hospital/Clinical College of Fujian Medical University, 156 Xihuanbei Road, Fuzhou, Fujian 350025, P.R. China

E-mail: fhlan2013@163.com

Key words: fragile $\mathrm{X}$ mental retardation 1, alternative splicing, cloning-sequencing, ratio of nonsynonymous substitutions per nonsynonymous site to synonymous substitutions per synonymous site

\section{Introduction}

Alternative splicing (AS) increases the complexity of eukaryotic gene expression and causes the diversification of protein function. Furthermore, 92-94\% of human multi-exon genes undergo alternative splicing $(1,2)$. Fragile $\mathrm{X}$ mental retardation 1 gene $(F M R I)$ is associated with fragile $\mathrm{X}$ syndrome (FXS). The gene is located on chromosome Xq27.3, is $38 \mathrm{~kb}$ long, and consists of 17 exons and 16 introns. FMRI AS gene expression has been observed in a number of different human and mouse tissues (3-6). Previous research has suggested that AS of the FMRI gene involves the inclusion or exclusion of exons 12 and 14 , and the selection of 3 ' splice sites in exons 15 and 17, which may generate up to 24 different mature transcripts. To date, 20 spliced isoforms (ISO; 1-20) have been reported $(3,4,6,7)$.

Spliced isoforms that are abundantly expressed are more likely to affect gene activity and patient physiology, compared with those that are expressed in low abundance (8). Analysis of the primary splice variant(s) that are expressed in different tissues and cell types is required, in order to understand the functions of alternatively spliced genes. Using reverse transcription-polymerase chain reaction (RT-PCR) and western blotting, a number of different alternatively spliced products have been observed in FMRl gene expression, in numerous types of tissue and cell lines. However, to the best of our knowledge, the proportion of spliced isoforms, expressed in different types of tissues and cells (3-7), has not been investigated. ISO7 (lacking exon 12) was found to be the predominant isoform expressed in COS cells and in lymphoblasts. By contrast, ISO1 (containing all exons) was not detected in any cell types, suggesting that ISO1 expression is very rare or absent in COS cells (7). Studies have demonstrated that ISO13 (containing the first splicing acceptor site in exon 17) is the dominant isoform in human heart, spleen, liver, kidney and fetal cerebral cortex tissues. ISO13 accounted for approximately $40 \%$ of all spliced isoforms in human fetal brain neurons, whereas other isoforms, including ISO1, were relatively rare $(9,10)$. ISO8 (lacking exon 12 and containing the second splicing acceptor site in exon 15) is dominant in human adult cerebral cortex tissue, in contrast with that of human fetal cerebral cortex tissue (10). These data suggest that the predominant splice 
variants vary between different types of tissues and cells. Therefore, analyses of FMRI gene AS profiles are required in order to further understand FXS pathogenesis, in particular in FXS-affected tissues, such as the brain and testis.

The FMRl gene AS site is located at $\sim 700 \mathrm{bp}$. The difference in size between alternatively spliced products is small. It is, therefore, difficult to distinguished AS sites using routine RT-PCR or northern blotting (10). A combined method, using RT-PCR and cloning, has been developed in order to detect alternatively spliced mRNA, and to analyze the splicing pattern of transcripts and the proportion of different alternatively spliced products $(9,11,12)$. In the present study, in order to analyze the AS expression of FMRI gene, a combined method, cloning and sequencing of the entire coding sequence of the FMR1 gene by means of TA cloning, was conducted in human peripheral blood, and brain and testis tissues.

\section{Materials and methods}

RT-PCR of the FMR1 gene from human peripheral blood. The present study was approved by the ethics committee of Fuzhou General Hospital (Fuzhou, China). Peripheral blood was collected from four healthy individuals following attainment of written informed consent and total RNA was extracted using an RNA isolation kit (Qiagen, Hilden, Germany). Total RNA (800 ng) was reverse transcribed using reverse transcriptase (Invitrogen, Life Technologies, Carlsbad, CA, USA). A 2070 bp fragment consisting of the entire coding region of the FMR I cDNA was amplified using the following primers: Forward: 5'-TCGAGCGCCCGCAGCCCACCTCT-3', and reverse: 5'-TGCCCTGTGCCATCTTGCCTACTATTT-3'. The PCR mixture $(50 \mu \mathrm{l})$ consisted of cDNA $(2 \mu \mathrm{l})$ and Taq DNA polymerase $(5 \mathrm{U})$. PCR conditions were as follows: Initial denaturation at $94^{\circ} \mathrm{C}$ for $5 \mathrm{~min}, 30$ cycles of denaturation at $94^{\circ} \mathrm{C}$ for $30 \mathrm{sec}$, annealing at $72^{\circ} \mathrm{C}$ for $3 \mathrm{~min}$ and extension at $72^{\circ} \mathrm{C}$ for $7 \mathrm{~min}$. All RT-PCR products were electrophoresed on $1 \%$ agarose gels (Sangon Biotech, Shanghai, China) and extracted using a QIAquick gel extraction kit (Qiagen).

RT-PCR of the FMR1 gene from human brain and testis $c D N A$. Human brain and testis QUICK-Clone cDNA was purchased from Clontech Laboratories, Inc. (Mountainview, CA, USA). Human whole brain and testis RNA was pooled from 8 and 39 healthy male Caucasians, respectively, who had died unexpectedly. The cDNA was used in order to amplify the entire coding region of the FMRl gene, as described for the human peripheral blood.

$T$ cloning-sequencing of RT-PCR products. Purified long chain RT-PCR products were ligated to pGEM-T Easy vector using an pGEM-T Easy vector system (Promega Corporation, Madison, WI, USA), according to the manufacturer's instructions. Subsequently, XL1-blue cells were transformed and the recombinants were selected on Luria-Bertani broth/ampicillin/isopropyl $\beta$-D-1-thiogalactop yranoside/X-Gal plates (all Sangon Biotech). Recombinants were identified using restrictive digestion with EcoRI and sent to a Shanghai-based company (Sangon Biotech), in order to sequence the inserts with Sanger sequencing.
Evolutionary selection pressure analysis of the FMRI exons. Vertebrate genome sequences (total 46, including human) were used for selection pressure analysis of the FMRl gene exons. Genome alignment information used in the present study was obtained from the UCSC (http://genome.ucsc.edu/). Vertebrate genome alignments (total 45) with human for coding DNA sequence (CDS) regions were obtained from ftp://hgdownload. cse.ucsc.edu/ (13). The aligned exon sequences (45 vertebrate genome alignments with human for CDS regions) were downloaded from the UCSC genome browser. Aligned exon sequences were then manually corrected according to the protein alignment (exon lengths were in multiples of three) and multiple sequence alignment analysis was then performed using MEGA 5.0 (14). Subsequently, the ratio of nonsynonymous substitutions per nonsynonymous site $(\mathrm{dN})$ to synonymous substitutions per synonymous site $(\mathrm{dS} ; \mathrm{dN} / \mathrm{dS})$ was estimated using the on-line DataMonkey package with likelihood-based methods (http://www.datamonkey.org), in order to measure selection pressure on amino acid sequences (15). A conservative single-likelihood ancestor-counting (SLAC) method was then conducted (16).

\section{Results}

Profiles of alternatively spliced products of the FMRI gene in human tissues. Recombinant products were randomly selected, with 71, 100 and 100 from RT-PCR products of human peripheral blood, brain and testis, respectively. Sequencing results indicated that 20 different spliced products of the FMR 1 gene were identified among the 271 recombinants (Fig. 1); 20 isoforms were identified that were not entirely consistent with those previously reported (3-6). Nomenclature in Fig. 1 is derived from that of Sittler et al (7). Six spliced products, ISO2, ISO4, ISO5, ISO6, ISO11 and ISO16, were not observed in the present study, suggesting that these six isoforms may be expressed in low levels in human peripheral blood, and brain and testis tissues.

ISO21-23 and ISO26 were only identified in peripheral blood sequences, whereas ISO24 and ISO25 were only observed in testis and brain sequences. ISO21 and ISO22 adopt different combinations to those splicing patterns in previous studies $(7,9)$. Furthermore, they may be included in the predicted 24 products (Fig. 1). ISO21, skipping exon 14, with the first splicing acceptor site in exon 15 and the second splicing acceptor site in selected exon 17, may encode a truncated polypeptide consisting of 439 amino acid residues with a different carboxyl terminus, as compared with the reference protein sequence of human FMRI (GenBank ID: AAB28395.2). ISO22, skipping exon 12, may encode a truncated polypeptide of 428 amino acid residues with a different carboxyl terminus. ISO23-26 were the result of the four novel splicing sites, whose sequences have been deposited in the GenBank database. ISO23 (GenBank ID: KC774029), skipping exons 11 and 12, with the second splicing acceptor site of selected exon 17, may encode a truncated polypeptide of 544 amino acid residues with a different carboxyl terminus. ISO24 (GenBank ID: KF856234), with the second splicing acceptor site of selected exon 15, may encode a truncated polypeptide of 554 amino acid residues without changing 


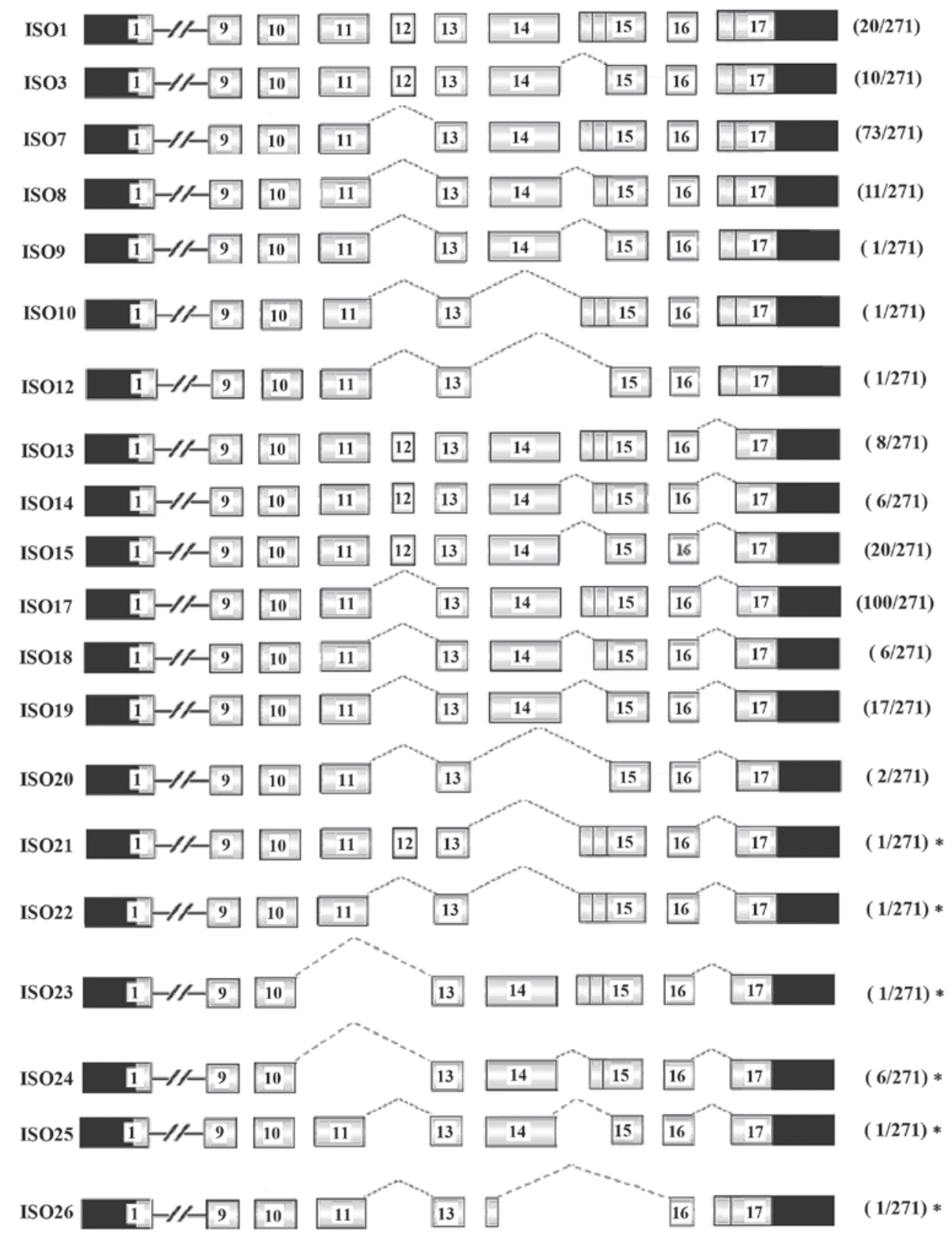

Figure 1. Alternatively spliced products of the FMRI gene in human peripheral blood cells, and brain and testis tissues. Black boxes represent non-coding regions. In brackets the numerator represents the detected spliced products of the corresponding ISO, and the denominator represents the total number of ISO. The dotted lines represent the different selected splicing sites. Novel splicing products are marked with an asterisk. ISO, isoform.

the open reading frame. ISO25 (GenBank ID: KC774027), with the novel splicing acceptor site of exon 15 and the selected third downstream site, may encode a polypeptide of 566 amino acid residues without changing the open reading frame. ISO26 (GenBank ID: KC774028), skipping exons 12 and 15, with a novel splicing donor site of exon 14 and a novel splicing acceptor site of selected exon 16, may encode a truncated polypeptide of 418 amino acid residues with a different carboxyl terminus (Fig. 2).

The results of the present study suggested that $\sim 50 \%$ of spliced isoforms are expressed at low levels, including those that have previously been reported. For example, only one spliced product of the following were observed: ISO9, 10 and 12, and the novel isoforms, ISO21-23, 25 and 26.
Spliced products containing the full-length coding sequence (ISO1) accounted for a low proportion of isoforms (7.38\%; 20/271). In particular, in sequences derived from brain tissue samples, ISO1 was detected once (1\%; Fig. 3).

Alternatively spliced products lacking exon 12 were predominant. The results of the analyses suggested that the following isoforms were the most abundantly expressed: ISO7, lacking exon 12 , and ISO17, lacking exon 12, with the second splicing acceptor site of exon 17 selected. However, the relative abundance of the these spliced products varied between tissues. In the brain, ISO17 was the predominant isoform (54\%), whilst in the testis, ISO7 was the predominant isoform (Fig. 3). In the brain, $99 \%$ of the isoforms lacked exon 12, and ISO1, 
Table I. Analysis of dN/dS ratios for 17 exons in FMRl gene.

\begin{tabular}{ll}
\hline Exon & $\mathrm{dN} / \mathrm{dS}$ \\
\hline Constitutive exons & 0.067 \\
Exon 1 & 0.153 \\
Exon 2 & 0.058 \\
Exon 3 & 0.081 \\
Exon 4 & 0.031 \\
Exon 5 & 0.041 \\
Exon 6 & 0.112 \\
Exon 7 & 0.079 \\
Exon 8 & 0.018 \\
Exon 9 & 0.061 \\
Exon 10 & 0.066 \\
Exon 13 & 0.054 \\
Alternative splicing exons & 0.410 \\
Exon 11 & 0.257 \\
Exon 12 & 1.735 \\
Exon 14 & 0.115 \\
Exon 15 & 0.092 \\
Exon 16 & 0.097 \\
Exon 17 & 0.165 \\
\hline
\end{tabular}

Exon sequences were analyzed using a single-likelihood ancestor-counting method using the DataMonkey package. dN/dS, ratio of nonsynonymous substitutions per nonsynonymous site (dN) to synonymous substitutions per synonymous site (dS) at each pre-aligned exon sequences; FMR1, Fragile X mental retardation 1.

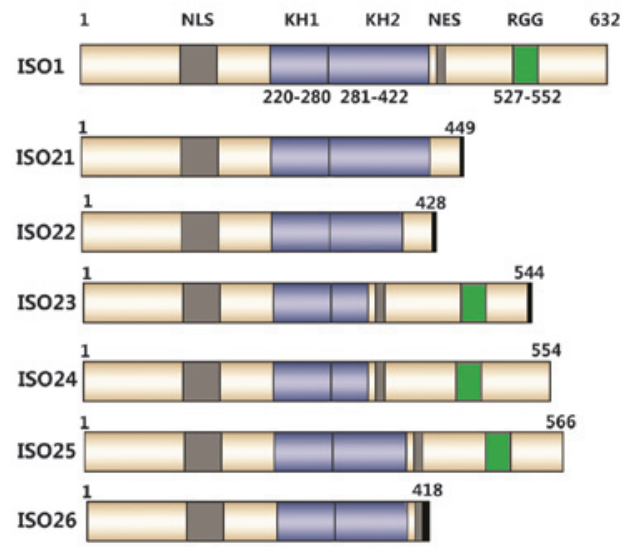

Figure 2. Predicted coding products of ISO1 and six novel splicing transcripts (ISO21-ISO26). ISO1 represents the FMR1 gene coding product with the full-length coding sequence, comprising NLS, KH1, KH2, NES and RGG. Numbers represent the location of amino acids. The black bar represents the carboxyl terminus of the premature termination codon. ISO, isoform; FMRI, fragile X mental retardation 1; NLS, nuclear localization signal; NES, nuclear export signal; $\mathrm{KH}, \mathrm{K}$ homology domain; RGG, arginine-glycine-rich region.

which consists of the entire coding sequence was detected only once.

Exon 12 may be under strong positive selection. According to the splicing pattern observed in the human FMR1 gene,
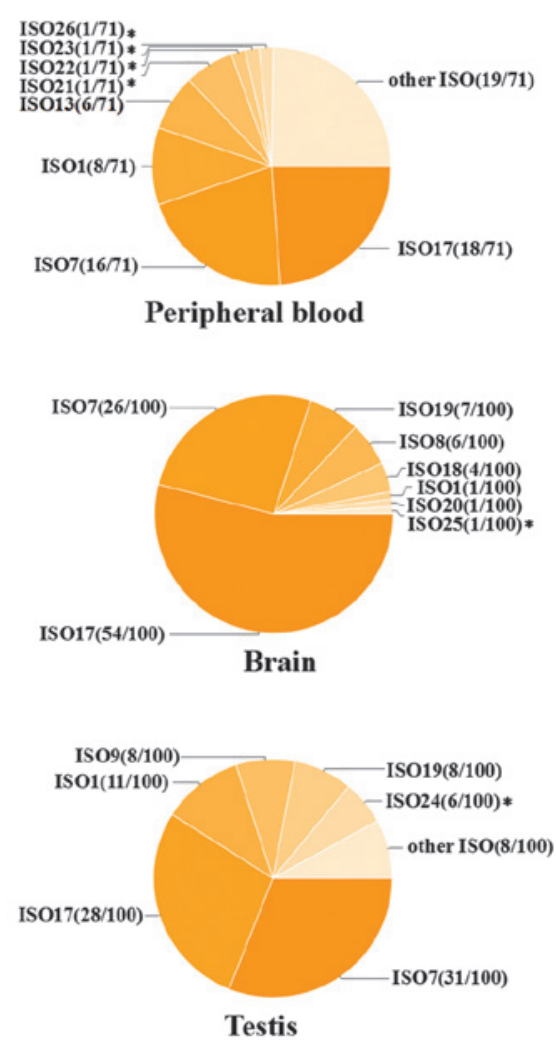

Figure 3. Expression of alternatively spliced products. Fractions in parentheses represent the approximate abundance of spliced products, in which the numerator represents the number of a particular spliced product and the denominator represents the total spliced transcripts. Novel splicing products are marked with an asterisk. ISO, isoform.

exons were classified into AS exons (exons 11-12, 14-17) and constitutive exons (exons 1-10,13). The results of an SLAC analysis of 17 pre-aligned exon sequences suggested that the mean $\mathrm{dN} / \mathrm{dS}$ value for AS exons was 0.410 , which was significantly higher than that for the constitutive exons $(0.067)$. The results demonstrated that the $\mathrm{dN} / \mathrm{dS}$ ratio of exon 12 was $>1$ $(\mathrm{dN} / \mathrm{dS}=1.735)$, whilst that of all other exons was $<1$ (Table I).

\section{Discussion}

In the present study, 20 types of alternatively spliced products were detected, six of which were previously unreported $(4-6,9,17,18)$. Among these six alternatively spliced products, four novel splicing patterns were identified, including an alternative 5 ' splice site, two alternative 3 ' splice sites and an exon skipping site. In addition, six previously reported spliced products were not observed in the present study. These results indicate that the AS of the FMRI gene may be more complex than previously understood.

In accordance with previous reports (7,9), ISO1 was not the most abundant isoform observed in the peripheral blood cells, and brain and testis tissues $(7.38 \% ; 20 / 271)$. Skipping of exon 14 has been found to affect the subcellular localization of the FMR protein (7). This suggests that AS may be involved in the functioning and regulation of the FMRI gene. More abundant splice variants are more likely to be associated with important gene functions, compared with those 
which are less abundant (8). In the present study, ISO7 and ISO17, lacking exon 12, were the dominant splicing products. In the brain, these isoforms together accounted for $80 \%$ of all isoforms (Fig. 3). Therefore, a lack of exon 12 may be involved in the physiological functioning of the FMRl gene.

FMR protein is an RNA-binding protein, abundant in the brain and testis, which selectively binds to its own mRNA and to $\sim 4 \%$ of brain mRNA, which is associated with neurodevelopment and synaptic plasticity $(19,20)$. It has been demonstrated that the discrepancy in FMRI transcripts between fetal and adult cerebral cortex tissue is associated with the presence of exon 12 , which is present in the fetal tissue but not in that of adults (10). Exon 12 lengthens the second $\mathrm{K}$ homology $(\mathrm{KH})$ domain, an FMRl RNA binding domain, which may influence the specificity or affinity of FMR protein RNA-binding (21). Exon 12 variants differentially interact with kissing complex RNA and exhibit a distinct RNA-binding specificity $(22,23)$. Furthermore, it has been reported that the I304N mutation located on the $\mathrm{KH} 2$ domain may lead to the expression of a serious FXS phenotype (24). Studies have shown that FMRI with a I304N mutation exhibited a tenfold average decrease in AUCK-containing RNA expression compared with that in wild-type FMRI (21). Therefore, AS products lacking exon 12 may shorten the second $\mathrm{KH} 2$ domain, which may influence the specificity and affinity of FMR protein RNA-binding and eventually target specific mRNA to regulate the corresponding protein expression. The results of the present study suggested that AS spliced products lacking exon 12 may be associated with the physiological functioning of FMRl gene.

Different types of selection pressure analyses have been conducted in order to predict the function of AS, such as $\mathrm{dN} / \mathrm{dS}$ (or $\mathrm{Ka} / \mathrm{Ks}$ ) against amino acid mutations, and $\mathrm{dS}$ (or $\mathrm{Ks}$ ) against mutations at synonymous sites, with the former being the most common $(25,26)$. A genome-wide selection pressure analysis in humans, chimpanzees, mice and rats has demonstrated that $\mathrm{Ka} / \mathrm{Ks}$ values were higher for AS exons compared with those for constitutive exons, implying that AS events may accelerate protein sequence evolution (27). In the present study, dN/dS values for FMRl exons demonstrated a similar pattern. Furthermore, exon 12 exhibited markedly high $(\geq 1) \mathrm{dN} / \mathrm{dS}$ values compared with that for other exons. This is in accordance with the results of a previous study that demonstrated higher dN/dS values for CD45 gene AS exons compared with those for constitutive exons, which suggests that the AS exons may be under positive selection (28). Therefore, exon 12, coding for part of the KH2 domain, may be under strong positive selection and associated with vertebrate evolution. Exon 12 consists of 63 nucleotides and, as this is a multiple of three, it is a 'modular' exon that may be inserted or removed from the transcripts without affecting the rest of the protein, which may facilitate AS functioning (29).

ISO24 is a novel spliced product that was detected in testis. It exhibited exon 11 skipping, which was also detected in ISO23 (Fig. 1). Exon 11 encodes part of the KH2 domain and consists of 135 nucleotides, and, therefore, like exon 12, may represent a 'modular' exon. The $\mathrm{dN} / \mathrm{dS}$ ratio analysis suggested that exon 11 exhibited higher value in AS exons, as compared with the other exons, except for exon 12 (Table I). The results of the present study indicated that the skipping of exon 11 may represent a functional AS event.
In conclusion, the results of the present study implied that AS spliced products lacking exon 12 may be associated with the physiological functioning the FMR 1 gene. Further investigation of the expression of isoforms ISO7 and ISO17 may help to improve the understanding of the structure and function of the FMR1 gene and the pathogenesis of FXS.

\section{Acknowledgements}

This study was supported by Key Scientific Projects of Fujian Province, P.R. China (grant no. 2010Y0045).

\section{References}

1. Johnson JM, Castle J, Garrett-Engele P, et al: Genome-wide survey of human alternative pre-mRNA splicing with exon junction microarrays. Science 302: 2141-2144, 2003.

2. Wang ET, Sandberg R, Luo S, et al: Alternative isoform regulation in human tissue transcriptomes. Nature 456: 470-476, 2008.

3. Ashley CT, Sutcliffe JS, Kunst CB, et al: Human and murine FMR-1: Alternative splicing and translational initiation downstream of the CGG-repeat. Nat Genet 4: 244-251, 1993.

4. Eichler EE, Richards S, Gibbs RA and Nelson DL: Fine structure of the human FMR1 gene. Hum Mol Genet 2: 1147-1153, 1993.

5. Verheij C, Bakker CE, de Graaff E, et al: Characterization and localization of the FMR-1 gene product associated with fragile X syndrome. Nature 363: 722-724, 1993.

6. Verkerk AJ, de Graaff E, De Boulle K, et al: Alternative splicing in the fragile $X$ gene FMR1. Hum Mol Genet 2: 399-404, 1993.

7. Sittler A, Devys D, Weber C and Mandel JL: Alternative splicing of exon 14 determines nuclear or cytoplasmic localisation of fmr1 protein isoforms. Human Mol Genet 5: 95-102, 1996.

8. Blencowe BJ: Alternative splicing: new insights from global analyses. Cell 126: 37-47, 2006.

9. Huang T, Li LY, Shen Y, Qin XB, Pang ZL and Wu GY: Alternative splicing of the FMR1 gene in human fetal brain neurons. Am J Med Genet 64: 252-255, 1996.

10. Ding J, Huang T, Li L, Fan Y and Shen Y: Alternative splicing of the FMR1 gene in human fetal tissues. Zhongguo Yi Xue Ke Xue Yuan Xue Bao 19: 241-246, 1997 (In Chinese).

11. Thackeray JR and Ganetzky B: Developmentally regulated alternative splicing generates a complex array of Drosophila para sodium channel isoforms. J Neurosci 14: 2569-2578, 1994.

12. Leparc GG and Mitra RD: A sensitive procedure to detect alternatively spliced mRNA in pooled-tissue samples. Nucleic Acids Res 35: e146, 2007.

13. Kuhn RM, Haussler D and Kent WJ: The UCSC genome browser and associated tools. Brief Bioinform 14: 144-161, 2013.

14. Tamura K, Peterson D, Peterson N, Stecher G, Nei M and Kumar S: MEGA5: Molecular evolutionary genetics analysis using maximum likelihood, evolutionary distance, and maximum parsimony methods. Mol Biol Evol 28: 2731-2739, 2011.

15. Poon AF, Frost SD and Pond SL: Detecting signatures of selection from DNA sequences using Datamonkey. Methods Mol Biol 537: 163-183, 2009.

16. Pond SL and Frost SD: Datamonkey: Rapid detection of selective pressure on individual sites of codon alignments. Bioinformatics 21: 2531-2533, 2005.

17. Koscielny G, Le Texier V, Gopalakrishnan C, et al: ASTD: The alternative splicing and transcript diversity database. Genomics 93: 213-220, 2009.

18. Takeda J, Suzuki Y, Sakate R, et al: H-DBAS: Human-transcriptome database for alternative splicing: Update 2010. Nucleic Acids Res 38: D86-D90, 2010.

19. Ashley CT Jr., Wilkinson KD, Reines D and Warren ST: FMR1 protein: Conserved RNP family domains and selective RNA binding. Science 262: 563-566, 1993.

20. Santoro MR, Bray SM and Warren ST: Molecular mechanisms of fragile X syndrome: A twenty-year perspective. Annu Rev Pathol 7: 219-245, 2012.

21. Ascano M Jr., Mukherjee N, Bandaru P, et al: FMRP targets distinct mRNA sequence elements to regulate protein expression. Nature 492: 382-386, 2012. 
22. Denman RB and Sung YJ: Species-specific and isoform-specific RNA binding of human and mouse fragile $\mathrm{X}$ mental retardation proteins. Biochem Biophys Res Commun 292: 1063-1069, 2002.

23. Xie W, Dolzhanskaya N, LaFauci G, Dobkin C and Denman RB Tissue and developmental regulation of fragile $X$ mental retardation 1 exon 12 and 15 isoforms. Neurobiol Dis 35: 52-62, 2009.

24. De Boulle K, Verkerk AJ, Reyniers E, et al: A point mutation in the FMR-1 gene associated with fragile $\mathrm{X}$ mental retardation. Nat Genet 3: 31-35, 1993.

25. Yang $Z$ and Nielsen R: Estimating synonymous and nonsynonymous substitution rates under realistic evolutionary models Mol Biol Evol 17: 32-43, 2000.
26. Xing $\mathrm{Y}$ and Lee C: Alternative splicing and RNA selection pressure - evolutionary consequences for eukaryotic genomes. Nat Rev Genet 7: 499-509, 2006.

27. Xing Y and Lee C: Evidence of functional selection pressure for alternative splicing events that accelerate evolution of protein subsequences. Proc Natl Acad Sci USA 102: 13526-13531, 2005.

28. Filip LC and Mundy NI: Rapid evolution by positive Darwinian selection in the extracellular domain of the abundant lymphocyte protein CD45 in primates. Mol Biol Evol 21: 1504-1511, 2004.

29. Xing Y and Lee CJ: Protein modularity of alternatively spliced exons is associated with tissue-specific regulation of alternative splicing. PLoS Genet 1: e34, 2005. 